\title{
Clinical value and role of microRNA-29c-3p in sepsis-induced inflammation and cardiac dysfunction
}

\author{
Bingyu Zhang ${ }^{1}$, Lin Yu ${ }^{1}$ and Ying Sheng ${ }^{2^{*}}$
}

\begin{abstract}
Background: The goal of this study was to investigate the diagnostic value of miR-29c-3p in sepsis and its role in sepsis-induced inflammatory response and cardiac dysfunction.

Methods: Serum level of miR-29c-3p was detected by qRT-PCR. The ROC curve was used to evaluate the diagnostic value of miR-29c-3p for Sepsis. The cecal ligation and puncture method (CLP) was used to establish a rat sepsis model. To assess cardiac function, left ventricular systolic pressure (LVSP), left ventricular end diastolic pressure (LVEDP) and maximum rate of rise/fall of left ventricle pressure $\left( \pm \mathrm{d} p / \mathrm{d} t_{\max }\right)$ in different experimental groups were detected, and the serum cardiac troponin I (CTnl), creative kinase isoenzyme MB (CK-MB) were measured by ELISA. Meanwhile, TNF$a, I L-1 \beta$, and IL- 6 were detected by ELISA to assess the level of inflammatory response in animals.
\end{abstract}

Results: miR-29c-3p level was upregulated in sepsis patients. ROC curve revealed that miR-29c-3p had the ability to distinguish sepsis patients from healthy controls. Cardiac dysfunction and inflammation were observed in sepsis rat, which were characterized by the decrease of LVSP and $+\mathrm{d} p / \mathrm{d} t_{\text {max }}$, the increase of LVEDP, $-\mathrm{d} p / \mathrm{d} t_{\text {max' }}, \mathrm{CTnl}, \mathrm{CK}-\mathrm{MB}$, TNF- $a, I L-1 \beta, I L-6$. All effects were reversed by the injection of miR-29c-3p antagomir. Logistics regression analysis manifested miR-29c-3p is an independent factor in the occurrence of cardiac dysfunction in sepsis patients.

Conclusions: miR-29c-3p has potential as a biomarker for the diagnosis of sepsis, and inhibition of miR-29c-3p expression in animal models reduced sepsis-induced cardiac dysfunction and inflammatory response.

Keywords: Sepsis, miR-29c-3p, Diagnosis, Cardiac dysfunction, Inflammation

\section{Background}

In recent years, modern medical diagnosis and treatment technology has advanced by leaps and bounds, but sepsis is still an important death cause for patients in the intensive care unit (ICU). It is estimated that about 5.3 million people die from sepsis every year in the world [1]. The relevant studies on sepsis are still a research hotspot in the medical field. Sepsis 3.0 defines sepsis as

\footnotetext{
*Correspondence: SY_sying@163.com

${ }^{2}$ Department of Emergency and Critical Care Medicine, Shanghai

Pudong Hospital, Fudan University Pudong Medical Center, No. 2800, Gongwei Road, Pudong district, Shanghai 201399, China

Full list of author information is available at the end of the article
}

a life-threatening condition resulting from a dysregulation of the body's response to infection [2]. The essence of sepsis is the excessive inflammatory reaction of the body caused by infection [3]. However, due to its complex pathophysiological mechanism, it involves multiple links such as inflammation and immunity [4], and it often takes several days to complete bacteriological evidence $[1,5]$. Therefore, the diagnosis and treatment of sepsis is still difficult due to the lack of specificity of rapid diagnosis and treatment.

MiRNAs are a class of non-coding RNAs (ncRNAs) with gene regulation function and approximately 19-24 nucleotides in length [6, 7]. By recognizing and combing with the $3^{\prime}$-non-transcriptional regions (3'-UTR)

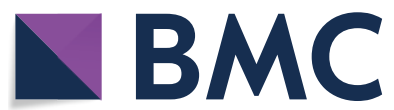

(c) The Author(s) 2021. Open Access This article is licensed under a Creative Commons Attribution 4.0 International License, which permits use, sharing, adaptation, distribution and reproduction in any medium or format, as long as you give appropriate credit to the original author(s) and the source, provide a link to the Creative Commons licence, and indicate if changes were made. The images or other third party material in this article are included in the article's Creative Commons licence, unless indicated otherwise in a credit line to the material. If material is not included in the article's Creative Commons licence and your intended use is not permitted by statutory regulation or exceeds the permitted use, you will need to obtain permission directly from the copyright holder. To view a copy of this licence, visit http://creativecommons.org/licenses/by/4.0/. The Creative Commons Public Domain Dedication waiver (http://creativeco mmons.org/publicdomain/zero/1.0/) applies to the data made available in this article, unless otherwise stated in a credit line to the data. 
of target mRNA, miRNAs guide the silencing complex to degrade mRNA or block its translation, thus affecting the expression of proteins in the next step [8]. Many miRNAs have been found to have specific diagnostic value in certain diseases. For example, in the myocardial cells of the sepsis mouse model, miR-223 expression was significantly down-regulated, and the further deletion of miR-223 aggravated the cardiac insufficiency and inflammatory response of the sepsis mice [9]. miR29c-3p belongs to the miR-29 family and was elevated in fibroblasts and VSMCs [10]. A recent study showed abnormally high miR-29c-3p in inflammatory diseases, such as adult-onset Still' s disease (AOSD) patients [11]. Considering that there are relatively few studies on the relationship between miR-29c-3p and sepsis, the specific mechanism remains to be explored.

In this present study, serum miR-29c-3p in sepsis patients has been detected and the clinical value of miR$29 c-3 p$ as a diagnostic marker of sepsis has also been assessed. At the same time, we further evaluated the effects of sepsis on cardiac function and inflammatory response in rats by establishing sepsis rat model.

\section{Materials and methods Participants and samples}

The study has been authorized by the Ethics Committee of Gongli Hospital of Pudong New Area of Shanghai, and each participant and his/her family members have been informed and signed a written informed consent. 86 sepsis patients admitted to the ICU of this hospital were recruited and the diagnostic criteria for sepsis follow the international diagnostic criteria for Sepsis 3.0 issued by SCCM/ESICM in 2016 [12]. Excluded participants met the following criteria: patients with chronic diseases, hematologic disease, and malignant tumors; people with congenital immune deficiency; pregnant or lactating women. Besides, 85 healthy people undergoing health examination in the same period were selected as the healthy controls, who had no major diseases or other abnormalities. Venous bloods were gathered from patients within $24 \mathrm{~h}$ of admission to the ICU and the APACHE-II score and SOFA score were estimated. The general clinical and clinicopathological indicators of the participants were collected and recorded.

\section{Construction of sepsis rat model}

Experiments involving animals were allowed by the Medical Ethics Committee of Gongli Hospital of Pudong New Area of Shanghai and were followed with the guidelines for the care and use of laboratory animals. 40 adult male SD rats weighing $250 \mathrm{~g}$ to $300 \mathrm{~g}$ were collected from Shanghai Animal Center. Before the experiment, they lived under suitable conditions $\left(23 \pm 1{ }^{\circ} \mathrm{C}\right.$, light/ dark cycle) and were allowed to eat and drink freely. As mentioned earlier, cecal ligation and puncture (CLP) was used to replicate the animal model of sepsis [13]. First, pentobarbital sodium $(50 \mathrm{mg} / \mathrm{kg})$ was used to anesthetize rats. A $2 \mathrm{~cm}$ surgical incision was made in the medioventral line of the rats, and the cecum was located after incision of the skin. Next, $1 / 3$ of the cecum was ligated with line 3 , and 3 punctures were performed with the needle of the No. 18 syringe at the center of the ligation end, while avoiding damage to blood vessels. Finally, the cecum was placed in the abdominal cavity and the skin was sutured with sutures 4 . Subcutaneously injected normal saline $(50 \mathrm{~mL} / \mathrm{kg})$ immediately after the operation for antishock. The control group received the same surgical treatment without CLP.

\section{Grouping and treatment}

SD rats were divided into control group (without CLPtreatment, injected with normal saline), CLP group (with CLP-treatment, injected with normal saline), miR-29c-3p $\mathrm{NC}$ group (with CLP-treatment, $10 \mu \mathrm{g}$ miR-29c-3p NC was injected intravenously $24 \mathrm{~h}$ before surgery) and miR29c-3p antagomir group (with CLP-treatment, $10 \mu \mathrm{g}$ miR-29c-3p antagomir was injected intravenously $24 \mathrm{~h}$ before surgery) with 10 rats in each group. The miR29c-3p NC and antagomir used in this study were synthesized and provided by GenePharma (Shanghai, China).

\section{Measurement of hemodynamic indexes}

The hemodynamic parameters of rats in each group including left ventricular systolic pressure (LVSP), left ventricular and end-diastolic pressure (LVEDP) and maximum rate of change in left ventricular pressure $( \pm \mathrm{d} p /$ $\mathrm{d} t_{\max }$ ) were monitored by MFLab 3.01 software on FDP-1 HRV and BRS system to evaluate the cardiac function of model rats.

\section{Detection of cardiac function and inflammatory indexes}

The venous blood of rats was collected for the detection of biochemical indexes. Serum level of cardiac troponin I (cTnI) and creative kinase isoenzyme MB (CK-MB), as well as tumor necrosis factor $\alpha$ (TNF- $\alpha)$, interleukin 6 (IL-6), interleukin $1 \beta$ (IL-1 $\beta$ ) were measured by Enzymelinked immunosorbent assay (ELISA) to evaluate the cardiac function and inflammatory response of rats in each group.

\section{Detection of miR-29c-3p expression levels}

qRT-PCR method detected the level of miR-29c-3p. RNAs were extracted from serum using TRIzol, and the RNAs were reverse transcribed into cDNA by a Prime Script ${ }^{\mathrm{TM}} \mathrm{RT}$ reagent Kit based on the product specification. Subsequently, PCR analysis was carried out using 
miScript SYBR ${ }^{\circledR}$ Green PCR kit on the PCR System. U6 was defined as internal reference, and the level of miR$29 \mathrm{c}-3 \mathrm{p}$ was calculated by normalization of U6 in $2^{-\Delta \Delta \mathrm{Ct}}$ methods.

\section{Statistical analysis}

SPSS and GraphPad Prism were used for statistical analysis. The student $t$-test or one-way ANOVA was used for comparison between groups, and the Chi-square test was used for comparison of classified variables.

Table 1 Clinical characteristics of the subjects

\begin{tabular}{llll}
\hline Parameter & \multicolumn{2}{l}{ Subjects $(\boldsymbol{N}=\mathbf{1 7 1})$} & $P$ value \\
\cline { 2 - 3 } & $\begin{array}{l}\text { Health } \\
\text { individuals } \\
(\boldsymbol{n}=\mathbf{8 5})\end{array}$ & $\begin{array}{l}\text { Sepsis patients } \\
(\boldsymbol{n}=\mathbf{8 6})\end{array}$ & \\
\hline Age (year) & $57.18 \pm 10.82$ & $54.56 \pm 12.91$ & 0.153 \\
Gender (male/female) & $40 / 45$ & $38 / 48$ & 0.706 \\
BMI (kg/m $\left.{ }^{2}\right)$ & $20.95 \pm 2.92$ & $21.83 \pm 2.97$ & 0.053 \\
Scr $(\mathrm{mg} / \mathrm{dL})$ & $1.03 \pm 0.28$ & $1.60 \pm 0.41$ & 0.000 \\
Albumin $(\mathrm{g} / \mathrm{L})$ & $42.51 \pm 5.84$ & $27.79 \pm 4.77$ & 0.000 \\
WBC $\left(\times 10^{9} / \mathrm{L}\right)$ & $7.21 \pm 1.51$ & $15.78 \pm 6.38$ & 0.000 \\
CRP $(\mathrm{mg} / \mathrm{L})$ & $5.51 \pm 2.80$ & $79.53 \pm 17.20$ & 0.000 \\
PCT $(\mathrm{ng} / \mathrm{mL})$ & $0.05 \pm 0.02$ & $11.10 \pm 4.00$ & 0.000 \\
APACHE $/ \mathrm{score}$ & - & $12.19 \pm 3.45$ & - \\
SOFA score & - & $5.05 \pm 1.60$ & - \\
\hline
\end{tabular}

Data are expressed as $\mathrm{n}$ or mean \pm standard deviation

$B M /$ body mass index, Scr serum creatinine, WBC white blood cell, $C R P C$-reactive protein, $P C T$ procalcitonin, APACHE acute physiology and chronic health evaluation, SOFA sequential organ failure assessment
The correlation between clinical indicators and miR$29 c-3 p$ level was analyzed by Pearson correlation coefficient. Logistic regression analysis was used to estimate the influence of different factors on cardiac dysfunction in sepsis patients. $P<0.05$ represented a significant difference.

\section{Results}

\section{Clinical characteristics of sepsis patients}

The study involved 171 participants, and the results of their clinical information comparison were shown in Table 1. There were no significant differences between the two groups in age, gender, and body mass index (BMI) $(P>0.05)$. The Scr, Albumin, WBC, CRP and PCT levels in sepsis patients were significantly different from those in healthy controls $(P<0.001)$. Besides, the APACHE II score and SOFA score of the sepsis patient were $(11.10 \pm 4.00)$ and $(5.05 \pm 1.60)$.

\section{Serum level of miR-29c-3p in sepsis patients}

qTR-PCR results showed that serum miR-29c-3p level in sepsis patients was significantly upregulated in comparison to healthy controls (Fig. $1 \mathrm{~A}, P<0.001$ ). Furthermore, serum miR-29c-3p level in sepsis patients with cardiac dysfunction was augmented remarkably than that in sepsis patients without cardiac dysfunction (Fig. 1B, $P<0.001$ ), suggesting that abnormal miR-29c-3p level was involved in the occurrence of sepsis, and high level of miR-29c-3p expression was associated with the condition of sepsis.

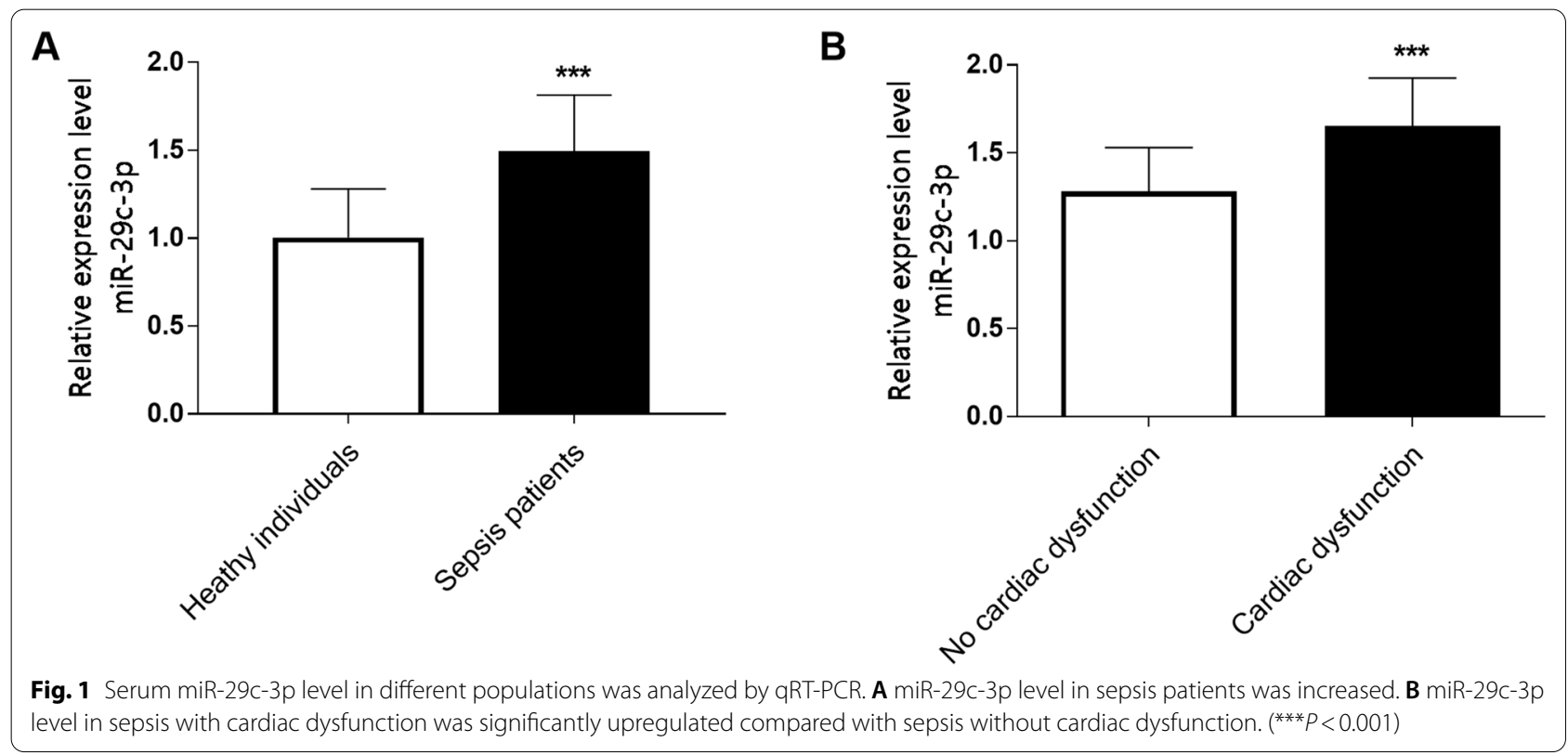




\section{Correlation analysis of miR-29c-3p and clinicopathological indicators of sepsis}

Pearson correlation coefficient analysis revealed that the levels of CRP and PCT, as well as APACHE II score and SOFA score were positively correlated with miR-29c-3p in sepsis patients (Table 2, $P<0.001$ ), indicating that the level of miR-29c-3p is positively correlated with the severity of sepsis.

\section{Analysis of the diagnostic value of miR-29c-3p in sepsis}

ROC curve was established to evaluate the diagnostic significance of miR-29c-3p in sepsis. In Fig. 2, it revealed that the AUC value was 0.872 with a sensitivity of $80.2 \%$ and specificity of $81.1 \%$, suggesting that serum miR$29 \mathrm{c}-3 \mathrm{p}$ is of high diagnostic value in sepsis.

\section{Association of miR-29c-3p levels with the occurrence of cardiac dysfunction}

Based on the cardiac function, patients were divided into normal cardiac function group $(n=37)$ and cardiac dysfunction group $(n=49)$. Subsequently, Logistics regression analysis evaluated the relationship between miR-29c-3p levels and the occurrence of cardiac dysfunction. It suggested that miR-29c-3p was an independent factor in the occurrence of cardiac dysfunction in sepsis patients (Table 3, OR $=3.905,95 \% \mathrm{CI}=1.410-10.817$, $P=0.009$ ).

Effects of miR-29c-3p on cardiac function in animal model The effect of miR-29c-3p on cardiac function in patients with sepsis was studied by constructing an animal model of sepsis in vitro. miR-29c-3p level in serum of CLP model rats was significantly increased (Fig. $3 \mathrm{~A}, P<0.001$ ), which showed a consistent trend with the results shown

Table 2 The relation of miR-29c-3p expression with the clinical variables

\begin{tabular}{lll}
\hline Parameters & Correlation $(\boldsymbol{r})$ & $\boldsymbol{P}$ value \\
\hline Age (year) & 0.045 & 0.682 \\
Gender (male/female) & 0.152 & 0.163 \\
$\mathrm{BMl}\left(\mathrm{kg} / \mathrm{m}^{2}\right)$ & 0.131 & 0.228 \\
$\mathrm{Scr}(\mathrm{mg} / \mathrm{dL})$ & 0.134 & 0.218 \\
Albumin $(\mathrm{g} / \mathrm{L})$ & 0.063 & 0.564 \\
WBC $\left(\times 10^{9} / \mathrm{L}\right)$ & 0.137 & 0.207 \\
CRP $(\mathrm{mg} / \mathrm{L})$ & 0.387 & 0.000 \\
PCT $(\mathrm{ng} / \mathrm{mL})$ & 0.474 & 0.000 \\
APACHE II score & 0.459 & 0.000 \\
SOFA score & 0.427 & 0.000 \\
\hline
\end{tabular}

$B M I$ body mass index, Scr serum creatinine, $W B C$ white blood cell, $C R P C$-reactive protein, $P C T$ procalcitonin, APACHE acute physiology and chronic health evaluation, SOFA sequential organ failure assessment

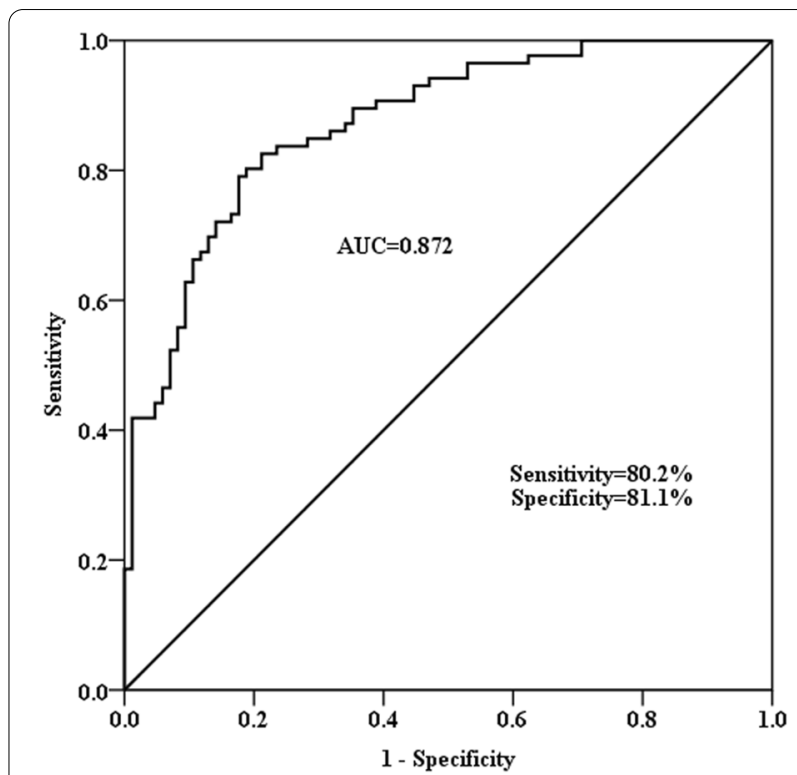

Fig. 2 ROC curve analysis

in Fig. 1. Besides, miR-29c-3p level in CLP rats was inhibited after intravenous administration of miR-29c-3p antagomir. Compared with the control group, LVSP and $+\mathrm{d} p / \mathrm{d} t_{\max }$ in the CLP group diminished, while LVEDP and $-\mathrm{d} p / \mathrm{d} t_{\max }$ enhanced significantly, accompanied by the increase of serum cTnI and CK-MB in the rats after monitoring the cardiac function, which indicated that cardiac dysfunction occurred in the sepsis rat model (Fig. 3B-F, $P<0.001)$. Moreover, we also noted that inhibition of miR-29c-3p after the injection of miR-29c-3p antagomir significantly improved cardiac function in

Table 3 Association of different variables with the occurrence of cardiac dysfunction

\begin{tabular}{llll}
\hline Parameter & OR & $\mathbf{9 5 \%} \mathbf{C l}$ & P value \\
\hline Age (year) & 1.804 & $0.660-4.930$ & 0.250 \\
Gender (male/female) & 1.161 & $0.445-3.029$ & 0.761 \\
BMI $\left(\mathrm{kg} / \mathrm{m}^{2}\right)$ & 1.200 & $0.455-3.166$ & 0.713 \\
$\mathrm{SCr}(\mathrm{mg} / \mathrm{dL})$ & 1.051 & $0.381-2.897$ & 0.923 \\
Albumin $(\mathrm{g} / \mathrm{L})$ & 0.505 & $0.179-1.429$ & 0.198 \\
WBC $\left(\times 10^{9} / \mathrm{L}\right)$ & 1.590 & $0.607-4.166$ & 0.346 \\
CRP $(\mathrm{mg} / \mathrm{L})$ & 1.194 & $0.428-3.333$ & 0.735 \\
PCT $(\mathrm{ng} / \mathrm{mL})$ & 1.002 & $0.373-2.695$ & 0.996 \\
APACHE II score & 1.146 & $0.429-3.057$ & 0.786 \\
SOFA score & 1.775 & $0.581-5.420$ & 0.314 \\
MiR-29c-3p & 3.905 & $1.410-10.817$ & 0.009 \\
\hline
\end{tabular}

$B M I$ body mass index, Scr serum creatinine, WBC white blood cell, CRP C-reactive protein, $P C T$ procalcitonin, $A P A C H E$, acute physiology and chronic health evaluation, SOFA sequential organ failure assessment 

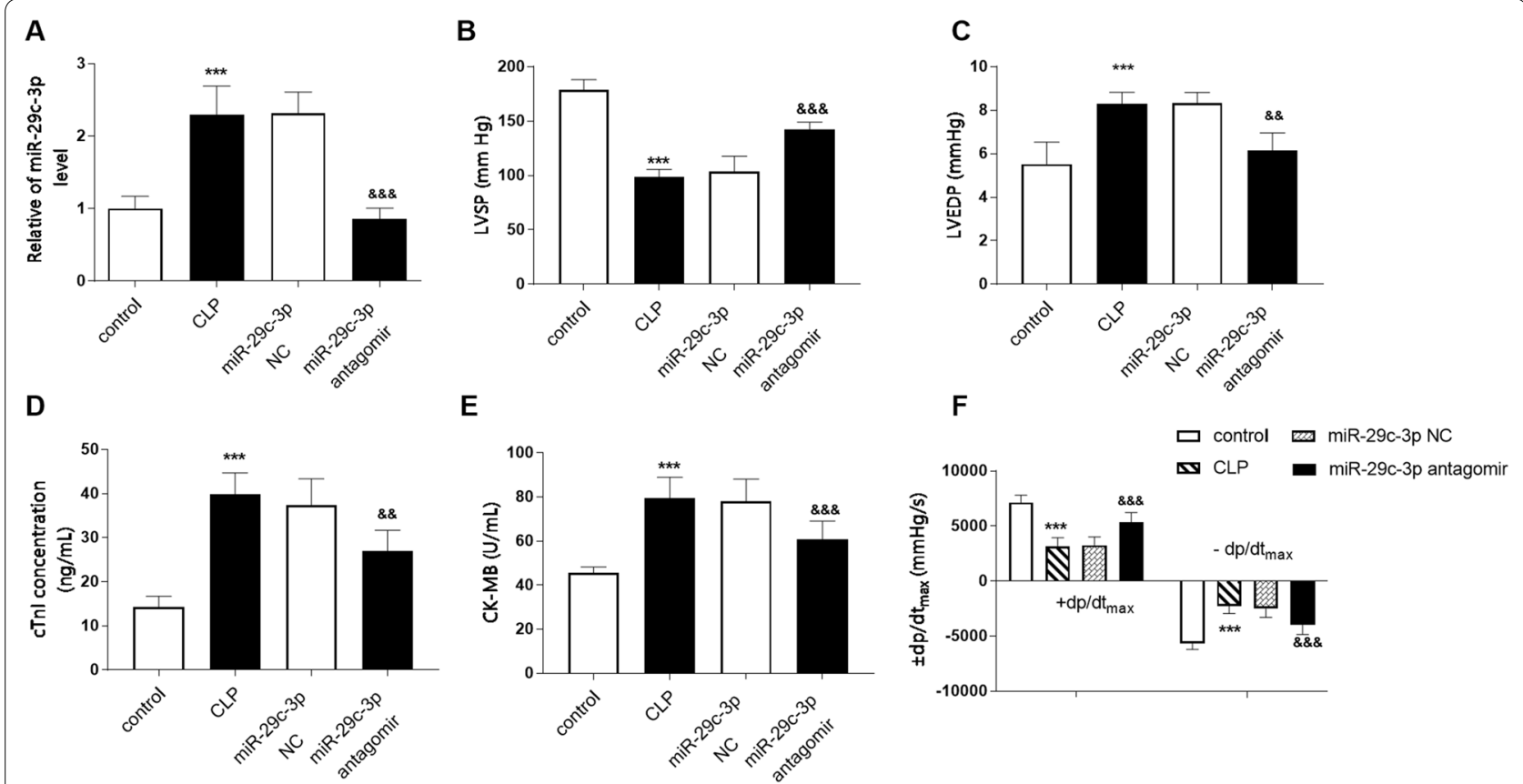

Fig. 3 Influences of miR-29c-3p on cardiac function in sepsis rat models. A Serum level of miR-29c-3p in different model groups. The changes of $\mathbf{B}$ LVSP, C LVEDP, $\mathbf{D} \subset T$ Tnl, E CK-MB and $\mathbf{F} \pm d p / \mathrm{d} t_{\max }$ in different model groups. (*** $P<0.001$ vs. control group, $\$ \$ p<0.001, \$ \$ p<0.01$ vs. CLP group)

CLP rats, which was mainly presented by increased LVSP and $+\mathrm{d} p / \mathrm{d} t_{\max }$, and decreased LVEDP and $-\mathrm{d} p / \mathrm{d} t_{\max }$, as well as the level of cTnI and CK-MB. In view of these results, we believe that miR-29c-3p is involved in the regulation of septic cardiac dysfunction.

Influences of miR-29c-3p on inflammation in sepsis rats

The levels of inflammatory indicators in serum were determined in sepsis rat models. As shown in Fig. 4, the levels of TNF- $\alpha$, IL- $1 \beta$ and IL- 6 were enhanced in the CLP group compared with the control group, while the downregulation of miR-29c-3p level could effectively reduce the levels of these inflammatory factors (Fig. 4AC, $P<0.01)$. These results suggested that inhibition of miR-29c-3p expression could down-regulate the level of sepsis induced inflammatory response.

\section{Discussion}

A rapidly developing systemic inflammatory response syndrome caused by infection is known as sepsis [14]. In clinical practice, sepsis can eventually develop into septic shock or multiple organ dysfunction syndrome if effective measures are not taken to control the early and continuous development of sepsis [15]. For the current
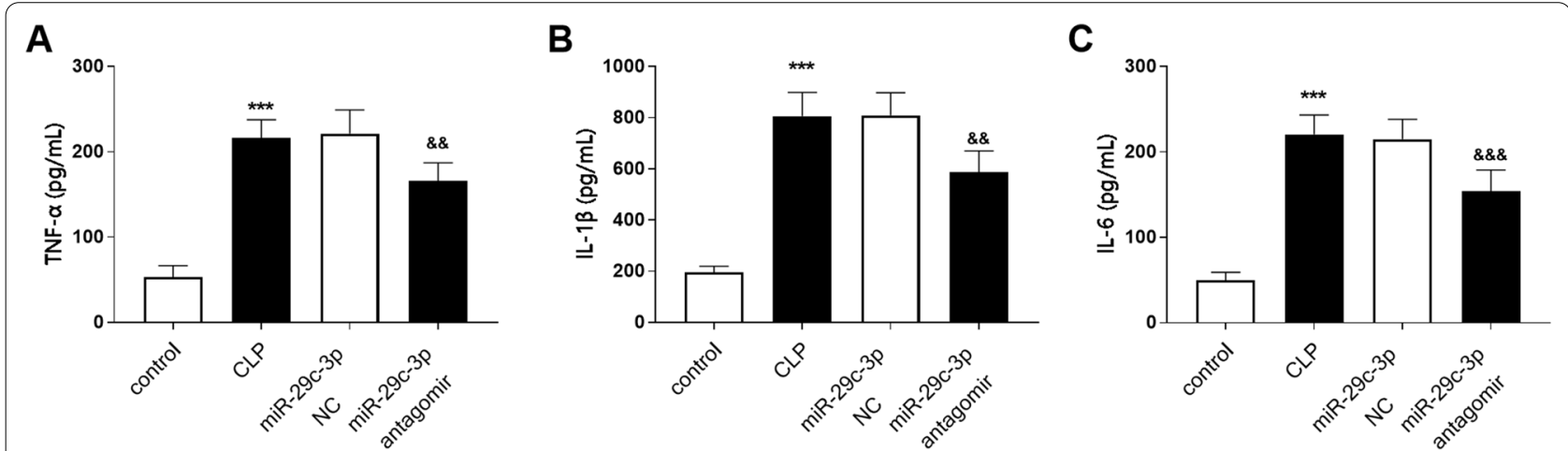

Fig. 4 Effects of miR-29c-3p on inflammatory cytokines in sepsis rats. Serum level of $\mathbf{A}$ TNF- $a, \mathbf{B} \| \mathrm{L}-1 \beta$, and $\mathbf{C} \| \mathrm{L}-6$ in sepsis rat models. ${ }^{* * *} P<0.001$ vs. control group, ${ }^{\$ \$} P<0.001,{ }^{\$} P<0.01$ vs. CLP group) 
diagnosis of sepsis, commonly used clinical biomarkers involve CRP, WBC, erythrocyte sedimentation rate (ESR), and procalcitonin (PCT) $[16,17]$. However, it is found that the above biomarkers are far from playing the expected role in practical application. Therefore, it is still necessary to continue to search for new and specific biomarkers to serve the clinical practice.

The ideal biomarker should have high sensitivity and specificity and should also be easy to obtain and quickly detect. miRNAs are widely present in body fluids, including blood and urine, and their highly stable characteristics in circulation make them capable of being used as biomarkers for clinical reference. As a tumor suppressor in the miR-29 family, miR-28c-3p, located at chromosome 1q32.3 [18], has been proved to be down-regulated in many solid tumors, such as gastric cancer, colorectal cancer, gallbladder cancer, etc. [19-21]. Fang et al's study showed that restraint of miR-29c-3p expression in laryngeal squamous cell carcinoma was significantly correlated with lymphatic metastasis and cancer typing [22]. In addition, Wu et al. reported that miR-29c-3p level was attenuated in patients with Alzheimer's disease. In our study, it was found that miR-29c-3p level was upregulated in sepsis patients, and it was closely related to the clinicopathological indicators of sepsis patients. Meanwhile, the ROC curve proved that the level of miR29c-3p had high sensitivity and specificity in the diagnosis of sepsis. Furthermore, after classifying sepsis patients according to whether cardiac dysfunction has occurred, we found that serum level of miR-29c-3p in sepsis patients with cardiac dysfunction was generally higher than that in sepsis patients without cardiac dysfunction. These findings suggested that high miR-29c-3p levels were correlated with the severity of sepsis.

According to statistics, sepsis patients with cardiac dysfunction have an increased mortality rate of $70 \%$ to $90 \%$ compared with patients who had no cardiac dysfunction [23]. The status of cardiovascular system and cardiac function in patients has always been the focus of basic clinical research during sepsis [24]. Studies have shown that hypoxia of the myocardium is a possible cause or sequelae of heart failure, it often occurs when coronary perfusion is reduced under high cardiac congestion pressure, including sepsis and high-altitude conditions [25]. Guo et al.s study exhibited that the level of miR-29c-3p in the right ventricle of hypoxic mice was significantly up-regulated over time [26]. Huang et al. showed that the level of miR-29c-3p was positively correlated with the degree of left ventricular hypertrophy in patients with systemic arterial hypertension [27]. Ye et al. revealed that miR-29c-3p promoted myocardial apoptosis by targeting MCL1 [28]. In this study, Logistics regression analysis proved that miR-29c-3p was an independent factor for cardiac dysfunction in sepsis patients. We evaluated the role of miR-29c-3p in sepsis-induced cardiac dysfunction by building a rat sepsis model. Our studies have shown that the serum miR-29c-3p expression of sepsis rat model was increased, and severe cardiac dysfunction appeared. However, it significantly improved the cardiac function of sepsis rats by suppressing miR-29c-3p expression. Our results are consistent with those of previous published studies. Therefore, we speculated that miR$29 c-3 p$ is involved in the regulation of cardiac function in sepsis rats. The relationship between miR-29 family and inflammatory response has been confirmed in many studies. For example, Chen et al. reported that the restoration of miR-29 levels in diabetic nephropathy inhibited the TGF- $\beta /$ Smad3 pathway, thereby reducing the accumulation of collagen matrix and inflammatory response [29]. Another study by Sun et al. has shown that miR29 c promotes inflammation in Type 2 diabetes mellitus by recruiting and activating circulating monocytes and macrophages [30]. Our study found that serum inflammatory cytokines were significantly enhanced in the sepsis rat model, while inhibition of miR-29c-3p expression decreased the level of inflammatory cytokines.

Based on the analysis of the above results, we think that the anti-inflammatory effect demonstrated by antagonistic miR-29c-3p may be related to its protective effect on cardiac function. However, the mechanism of miR$29 c-3 p$ regulating cardiac function and inflammatory response is still unknown. These tissues as the limitations of this study still need further exploration.

In general, serum miR-29c-3p level was increased in sepsis patients. And miR-29c-3p expression was up-regulated in sepsis patients with cardiac dysfunction than in sepsis patients without cardiac dysfunction. miR-29c-3p level has high clinical value for the diagnosis of sepsis. Animal model experiments have confirmed that inhibition of miR-29c-3p improved sepsis-induced cardiac dysfunction and inflammatory response.

\section{Abbreviations \\ AOSD: Adult-onset Still's disease; APACHE-II: Acute physiological and chronic health; BMI: Body mass index; CK-MB: Creative kinase isoenzyme MB; CLP: Cecal ligation and puncture; CRP: C-reactive protein; CTnl: Cardiac troponin I; ESR: Erythrocyte sedimentation rate; ICU: Intensive care unit; IL-6: Interleukin 6; IL-1 $\beta$ : Interleukin 1 $\beta$; LVSP: Left ventricular systolic pressure; LVEDP: Left ventricular end diastolic pressure; PCT: Procalcitonin; SOFA: Score and organ dysfunction; TNF-a: Tumor necrosis factor $a$; WBC: White blood cell; $3^{\prime}$-UTR: 3'-Non-transcriptional regions.}

\section{Acknowledgements}

Not applicable.

\section{Authors' contributions}

$\mathrm{BZ}$ and YS conceived and planned the study. All authors carried out the experiments, analyzed and interpreted the data. BZ contributed in writing the manuscript. All authors read and approved the final manuscript. 


\section{Funding}

This study was supported by grants from Key Discipline Construction Project of Pudong Health Bureau of Shanghai (Grant No. PWZxk2017-20).

\section{Availability of data and materials}

The datasets used and/or analysed during the current study are available from the corresponding author on reasonable request.

\section{Declarations}

\section{Ethics approval and consent to participate}

This study has been approved by the Ethics Committee of Gongli Hospital of Pudong New Area of Shanghai, and each participant and his/her family members have been informed and signed a written informed consent. All animal operations were conducted according to the guidelines for the care and use of laboratory animals and approved by the medical ethics committee of Gongli Hospital of Pudong New Area of Shanghai.

\section{Consent for publication}

Patients signed informed consent regarding publishing their data.

\section{Competing interests}

The authors declare that they have no competing interests.

\section{Author details}

${ }^{1}$ Department of Critical Care Medicine, Gongli Hospital of Pudong New Area of Shanghai, Shanghai 200135, China. ${ }^{2}$ Department of Emergency and Critical Care Medicine, Shanghai Pudong Hospital, Fudan University Pudong Medical Center, No. 2800, Gongwei Road, Pudong district, Shanghai 201399, China.

Received: 31 May 2021 Accepted: 29 June 2021

Published online: 10 August 2021

\section{References}

1. Fleischmann C, Scherag A, Adhikari NK, Hartog CS, Tsaganos T, Schlattmann $P$, et al. Assessment of global incidence and mortality of hospitaltreated sepsis. Current estimates and limitations. Am J Respir Crit Care Med. 2016;193(3):259-72.

2. Singer M, Deutschman CS, Seymour CW, Shankar-Hari M, Annane D, Bauer $M$, et al. The third international consensus definitions for sepsis and septic shock (sepsis-3). JAMA. 2016;315(8):801-10.

3. Dantes RB, Epstein L. Combatting sepsis: a public health perspective. Clin Infect Dis. 2018;67(8):1300-2.

4. Gentile LF, Cuenca AG, Efron PA, Ang D, Bihorac A, McKinley BA, et al. Persistent inflammation and immunosuppression: a common syndrome and new horizon for surgical intensive care. J Trauma Acute Care Surg. 2012;72(6):1491-501.

5. Teggert A, Datta H, Ali Z. Biomarkers for point-of-care diagnosis of sepsis. Micromachines. 2020;11(3):286.

6. Deiuliis JA. MicroRNAs as regulators of metabolic disease: pathophysiologic significance and emerging role as biomarkers and therapeutics. Int J Obes. 2016;40(1):88-101.

7. Tsitsiou E, Lindsay MA. microRNAs and the immune response. Curr Opin Pharmacol. 2009;9(4):514-20.

8. Bartel DP. MicroRNAs: genomics, biogenesis, mechanism, and function. Cell. 2004;116(2):281-97.

9. Wang $X$, Huang W, Yang $Y$, Wang $Y$, Peng T, Chang J, et al. Loss of duplexmiR-223 (5p and 3p) aggravates myocardial depression and mortality in polymicrobial sepsis. Biochim Biophys Acta. 2014;1842(5):701-11.

10. Ulrich V, Rotllan N, Araldi E, Luciano A, Skroblin P, Abonnenc M, et al. Chronic miR-29 antagonism promotes favorable plaque remodeling in atherosclerotic mice. EMBO Mol Med. 2016:8(6):643-53.

11. Hu Q, Gong W, Gu J, Geng G, LiT, Tian R, et al. Plasma microRNA profiles as a potential biomarker in differentiating adult-onset Still's disease from sepsis. Front Immunol. 2018;9:3099.
12. Seymour CW, Liu VX, Iwashyna TJ, Brunkhorst FM, Rea TD, Scherag A, et al. Assessment of clinical criteria for sepsis: for the third international consensus definitions for sepsis and septic shock (sepsis-3). JAMA. 2016;315(8):762-74

13. Dejager $\mathrm{L}$, Pinheiro I, Dejonckheere $\mathrm{E}$, Libert C. Cecal ligation and puncture: the gold standard model for polymicrobial sepsis? Trends Microbiol. 2011;19(4):198-208

14. Zou Q, Wen W, Zhang XC. Presepsin as a novel sepsis biomarker. World J Emerg Med. 2014:5(1):16-9.

15. Angus DC, van der Poll T. Severe sepsis and septic shock. N Engl J Med. 2013;369(9):840-51.

16. Jekarl DW, Lee S, Kim M, Kim Y, Woo SH, Lee WJ. Procalcitonin as a prognostic marker for sepsis based on SEPSIS-3. J Clin Lab Anal. 2019;33(9):e22996.

17. Raveendran AV, Kumar A, Gangadharan S. Biomarkers and newer laboratory investigations in the diagnosis of sepsis. J R Coll Physicians Edinb. 2019;49(3):207-16.

18. Luan J, Wang J, Su Q, Chen X, Jiang G, Xu X. Meta-analysis of the differentially expressed microRNA profiles in nasopharyngeal carcinoma. Oncotarget. 2016;7(9):10513-21.

19. Matsuo M, Nakada C, Tsukamoto Y, Noguchi T, Uchida T, Hijiya N, et al. MiR-29c is downregulated in gastric carcinomas and regulates cell proliferation by targeting RCC2. Mol Cancer. 2013;12:15.

20. Chen G, Zhou T, Li Y, Yu Z, Sun L. p53 target miR-29c-3p suppresses colon cancer cell invasion and migration through inhibition of PHLDB2. Biochem Biophys Res Commun. 2017;487(1):90-5.

21. Shu YJ, Bao RF, Jiang L, Wang Z, Wang XA, Zhang F, et al. MicroRNA$29 c-5 p$ suppresses gallbladder carcinoma progression by directly targeting CPEB4 and inhibiting the MAPK pathway. Cell Death Differ. 2017;24(3):445-57.

22. Fang $R$, Huang $Y$, Xie J, Zhang J, Ji X. Downregulation of miR-29c-3p is associated with a poor prognosis in patients with laryngeal squamous cell carcinoma. Diagn Pathol. 2019;14(1):109.

23. Parrillo JE, Parker MM, Natanson C, Suffredini AF, Danner RL, Cunnion $\mathrm{RE}$, et al. Septic shock in humans. Advances in the understanding of pathogenesis, cardiovascular dysfunction, and therapy. Ann Intern Med. 1990;113(3):227-42

24. Merx MW, Weber C. Sepsis and the heart. Circulation. 2007; 116(7):793-802.

25. Rabinovitch M, Guignabert C, Humbert M, Nicolls MR. Inflammation and immunity in the pathogenesis of pulmonary arterial hypertension. Circ Res. 2014;115(1):165-75.

26. Chouvarine P, Legchenko E, Geldner J, Riehle C, Hansmann G. Hypoxia drives cardiac miRNAs and inflammation in the right and left ventricle. J Mol Med. 2019;97(10):1427-38.

27. Huang Y, Tang S, Huang C, Chen J, Li J, Cai A, et al. Circulating miRNA29 family expression levels in patients with essential hypertension as potential markers for left ventricular hypertrophy. Clin Exp Hypertens. 2017:39(2):119-25

28. Ye Y, Hu Z, Lin Y, Zhang C, Perez-Polo JR. Downregulation of microRNA-29 by antisense inhibitors and a PPAR-gamma agonist protects against myocardial ischaemia-reperfusion injury. Cardiovasc Res. 2010;87(3):535-44.

29. Chen HY Zhong X, Huang XR, Meng XM, You Y, Chung AC, et al. MicroRNA-29b inhibits diabetic nephropathy in db/db mice. Mol Ther. 2014;22(4):842-53.

30. Sun Y, Zhou Y, Shi Y, Zhang Y, Liu K, Liang R, et al. Expression of miRNA-29 in pancreatic beta cells promotes inflammation and diabetes via TRAF3. Cell Rep. 2021;34(1):108576.

\section{Publisher's Note}

Springer Nature remains neutral with regard to jurisdictional claims in published maps and institutional affiliations. 